\title{
Etude du champ lointain de la double hétérostructure laser GalnAsSb/GaAlAsSb
}

Messanh A. MOHOU*, Milohum M. DZAGLI et Komlan S. GADEDJISSO-TOSSOU

Laboratoire de Physique des Composants à Semi-conducteurs (LPCS),

Département de Physique, Université de Lomé, FDS/UL- BP I515 Lomé - Togo

*Correspondance, courriel : mmagbeko@yahoo.fr

\section{Résumé}

La théorie électromagnétique de la double hétérostructure (DH) laser

$G a_{1-x} A I_{x} A s_{y} S b_{1-y} / G a_{1-x^{\prime}} / n_{x^{\prime}} A s_{y^{\prime}} S b_{1-y^{\prime}} / G_{1-x} A a_{x} A s_{y} S b_{1-y} \quad a$ été réalisée à partir des équations de Maxwell, Huyghens et Fraunhofer. Elle a permis d'établir l'expression du champ lointain dans le plan orthogonal à l'hétérojonction.

L'optimisation de la structure laser a permis d'établir les conditions pour un meilleur couplage de la DH laser avec la fibre optique.

En particulier, nous montrons que le champ perpendiculaire dépend de la différence d'indices $\Delta \mathrm{n}=\left(\mathrm{n}_{2}-\mathrm{n}_{1}\right)$.

Mots-clés : Double hétérostructure, Equations de Maxwell, Diode laser, champ lointain.

\section{Abstract}

Study of the far field of the double heterostructure laser GalnAsSb/GaAlAsSb

The electromagnetic theory of the double heterostructure (DH) laser $\mathrm{Ga}_{1-x} A \mathrm{I}_{x} A s_{y} S b_{1-y} / \mathrm{Ga}_{1-x} \cdot \mathrm{n}_{\mathrm{x}}, \mathrm{As}_{\mathrm{y}}, \mathrm{Sb}_{1-\mathrm{y}} / \mathrm{Ga}_{1-x} \mathrm{Al}_{\mathrm{x}} \mathrm{As} \mathrm{S}_{\mathrm{y}} \mathrm{Sb}_{1-y}$ has been realised using the Maxwell, Huyghens and Fraunhofer equations. This has permitted the establishment of an expression for the far field in the orthogonal plane of the heterojunction.

The optimization of the structure laser allowed the establishment the conditions for a better coupling of the DH laser with the optical fibres. 


$$
\text { Afrique SCIENCE 05(1)(2009) } 58 \text { - } 66
$$

In particular, we show that the perpendicular field depends on the gap of the index $\Delta \mathrm{n}=\left(\mathrm{n}_{2}-\mathrm{n}_{1}\right)$.

Keywords : Heterostructure, Maxwell equations, Diode laser, Far field.

\section{Introduction}

Les diodes lasers étudiées sont du type planar à ruban avec une cavité Fabry-Pérot, comme l'indique la figure 1 . Au cours des études précédentes [1], l'intérêt de ces diodes laser a été expliqué. II est montré [2-5] que de tels lasers présentent d'intéressantes applications.

L'expression du champ lointain dans le plan orthogonal à l'hétérojonction et la variation de l'écart des angles à mi-hauteur sur la courbe d'intensité en fonction de la composition x d'Aluminium constituent l'objet de cet article.

\section{Matériel et méthodes}

\section{2-1. Champ rayonné à Grande distance}

On définit le champ à grande distance comme étant le champ électromagnétique rayonné par la double hétérostructure laser à une distance $h$ très grande par rapport aux dimensions de la face de sortie.

La Figure $I$ représente la structure considérée, $\mathrm{n}_{2}$ représente l'indice de la couche active, $\mathrm{n}_{1}$ celui des couches de confinement. La Figure $\boldsymbol{l}$ illustre également les hypothèses faites pour le calcul de l'intensité du champ lointain.

Chaque point de la face de sortie (Figure $\prod$ du laser peut être considéré comme une source radiative dans le demi-espace $Z>0$.

D'après le principe de Huyghens, l'amplitude du champ électrique en un point d'observation A peut être considérée comme la somme des amplitudes au point A des vibrations émises par les sources élémentaires cohérentes localisées sur la face de sortie.

On se propose de calculer le champ électrique dans la direction perpendiculaire à la jonction plane au point $A(y, z)$ dans l'espace. 
Afrique SCIENCE 05(1)(2009) $58-66$

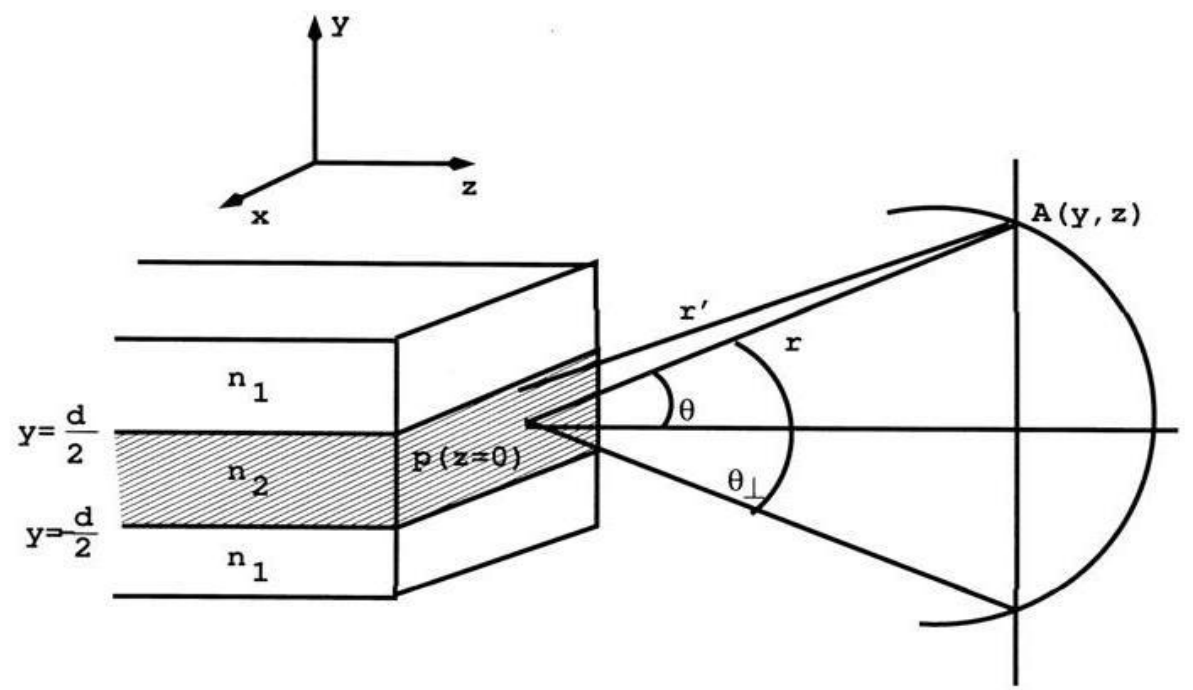

Figure 1 : Structure considérée

Compte tenu de la géométrie de la structure, l'équation du champ électrique en A s'écrit :

$$
\frac{\partial^{2}}{\partial \mathbf{y}^{2}} \mathbf{E}_{\mathbf{x}}+\frac{\partial^{2}}{\partial \mathbf{z}^{2}} \mathbf{E}_{\mathbf{x}}=\boldsymbol{\mu}_{0} \boldsymbol{\varepsilon}_{0} \frac{\partial^{2}}{\partial \mathbf{t}^{2}} \mathbf{E}_{\mathbf{x}}
$$

avec $\quad \mu_{0} \varepsilon_{0} c^{2}=1$

La méthode de la séparation des variables permet d'écrire si $E(y, z, t)=Y(y) Z(z)$ $\exp (j \omega t)$ :

$$
\frac{Y^{\prime \prime}}{Y}+\frac{Z^{\prime \prime}}{Z}=-\mu_{0} \varepsilon_{0} \omega^{2}=-K_{0}^{2}
$$

Si on introduit le paramètre $u$ en décomposant $K_{0}$ selon le schéma suivant : 


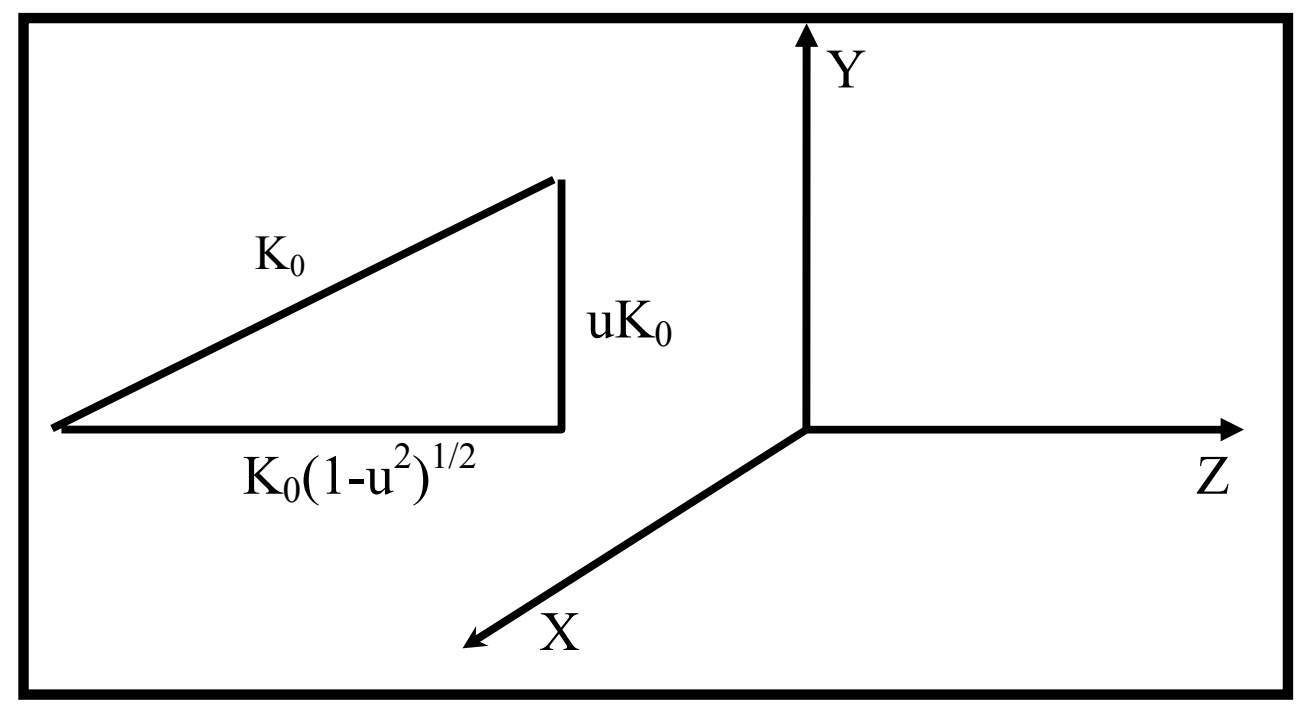

L'équation (2) devient :

$$
\frac{Y^{\prime \prime}}{Y}=-\frac{Z^{\prime \prime}}{Z}-K_{0}^{2}=-\mathbf{u}^{2} \mathbf{k}_{0}^{2}
$$

Les solutions suivant les directions $0 y$ et $0 z$ peuvent s'écrire :

$$
\begin{array}{r}
Y=A \exp \left(j u K_{0} y\right)+B \exp \left(-j u K_{0} y\right) \\
Z=C \exp \left[i\left(1-u^{2}\right)^{1 / 2} K_{0}^{2} z\right]+D \exp \left[-i\left(1-u^{2}\right)^{1 / 2} K_{0}^{2} z\right]
\end{array}
$$

où $A, B, C$ et $D$ sont supposés être des fonctions de $u$.

Afin de traduire le fait que u ne peut prendre le signe opposé, un seul terme doit être retenu dans les deux équations. Une solution particulière pour une valeur donnée de u peut se mettre sous la forme :

$$
E_{x}(y, z)=h(u) \exp \left(-j u K_{0} y\right) \exp \left[-j\left(1-u^{2}\right)^{1 / 2} K_{0} z\right]
$$

$\operatorname{arech}(u)=B D$

La solution générale est obtenue en intégrant sur toutes les valeurs de u 
Afrique SCIENCE 05(1)(2009) 58 - 66

$$
E_{x}(y, z)=\int_{-\infty}^{+\infty} h(u) \exp \left(-j u K_{0} y\right) \exp \left[-j\left(1-u^{2}\right)^{1 / 2} K_{0} z\right] d u
$$

Pour évaluer cette intégrale, nous utilisons la méthode de la descente rapide et celle de la phase stationnaire $[6,14]$.

Si nous posons: $y=r \sin \theta ; z=r \cos \theta$ et formulons l'hypothèse que la largeur électromagnétique de l'onde est faible comparée à la distance du point d'observation, alors l'équation (5) devient :

$$
E(\theta)=\left[\frac{K_{0}}{2 \pi \pi}\right]^{1 / 2} \exp (j \pi 4) \int E_{x}(y, 0) \exp \left[j K_{0} y \sin \theta\right] d y
$$

On voit donc que le champ à grande distance se déduit de la luminance sur la face de sortie par la transformée de Fourier.

Une telle équation est similaire au résultat de la théorie de la diffraction de Fraunhofer pour une onde plane qui arrive sur une ouverture dans un écran opaque sous un faible angle d'incidence. La variation relative de l'intensité du champ lointain est donnée par :

$$
\frac{I(\theta)}{I(0)}=\frac{|E(\theta)|^{2}}{|E(0)|^{2}}=\cos ^{2} \theta \frac{\left|\int_{-\infty}^{+\infty} E_{x}(y, 0) \exp \left(j K_{0} \sin \theta y\right) d y\right|^{2}}{\left|\int_{-\infty}^{+\infty} E_{x}(y, 0) d y\right|^{2}}
$$

En remplaçant $E_{x}(y, 0)$ par les expressions obtenues dans le cas d'une structure symétrique et tenant compte de la relation de dispersion relative à une structure symétrique qui s'écrit : $\tan \left(K_{\mathrm{y} 2} \mathrm{~d} / 2\right)=\mathrm{K}_{\mathrm{y} 1} / \mathrm{K}_{\mathrm{y} 2}$, on obtient :

$$
\frac{I(\theta)}{I(0)}=\cos ^{2} \theta\left[K_{y 2}^{2} K_{y 1} \frac{K_{y 1} \cos \left(K_{0} \frac{d}{2} \sin \theta\right)-K_{0} \sin \theta \sin \left(K_{0} \frac{d}{2} \sin \theta\right)}{\left(K_{y 2}^{2}-K_{0}^{2} \sin ^{2} \theta\right)\left(K_{y 1}^{2}+K_{0}^{2} \sin ^{2} \theta\right)}\right]^{2}
$$

Signalons parmi les méthodes de calcul du champ lointain, les travaux de Botez [13], Byer et al [15], Butler et al [16] et Itaya et al. [17]. 
63

$$
\text { Afrique SCIENCE 05(1)(2009) } 58-66
$$

L'équation trouvée est d'une manière générale similaire à l'expression (8) au terme d'obliquité $\cos \theta$ près. Dans cet article, nous ne prendrons pas en compte le facteur d'obliquité.

\section{Résultats et Discussion}

Nous présentons dans cette partie les résultats de nos calculs. L'étude théorique de la variation du champ lointain de la double hétérostructure laser $G a_{1-x} A I_{x} A s_{y} S b_{1-y} / G a_{1-x} / n_{x} A s_{y} S b_{1-y} / G a_{l-x} A l_{x} A s_{y} S b_{l-y}$ nécessite la connaissance des indices de réfraction des alliages. Les courbes présentées dans ce paragraphe sont obtenues à partir des données du Tableau 1.

Tableau 1 : Indice de réfraction à $300 \mathrm{~K}$

\begin{tabular}{|c|l|l|l|}
\hline \multirow{2}{*}{$\mathrm{Ga}_{0,83} \mathrm{In}_{0,17} \mathrm{As}_{0,15} \mathrm{Sb}_{0,85}$} & \multicolumn{3}{|c|}{$\mathrm{Ga}_{1-x} \mathrm{Al}_{\mathrm{x}} \mathrm{As}_{y} \mathrm{Sb}_{1-y}$} \\
\hline \multirow{3}{*}{3,72} & $y=2,4 \%$ & $y=3 \%$ & $y=4,5 \%$ \\
& $x=27 \%$ & $x=34 \%$ & $x=50 \%$ \\
& 3,63 & 3,59 & 3,50 \\
& & & \\
\hline
\end{tabular}

La Figure 2 illustre l'évolution du champ lointain dans le plan orthogonal à l'hétérojonction. Les valeurs de l'écart des angles à mi-hauteur sur la courbe d'intensité varient en fonction de la composition $x$ d'Aluminium. Pour $\mathrm{x}=50 \%, \theta_{\perp}$ $=69^{\circ}$ et n'est plus que de $40^{\circ}$ pour $x=27 \%$. Ceci veut dire qu'une valeur minimale de $x$ est nécessaire en vue d'obtenir une quantité de $\theta_{\perp}$ satisfaisante permettant d'améliorer l'efficacité du couplage de la DH laser avec la fibre optique.

L'étude de l'évolution de la largeur à mi-hauteur $\theta_{\perp}$ en fonction de l'indice de réfraction $\mathrm{n}_{\text {act }}$ de la couche active est représentée sur la figure 3 . L'indice de la couche de confinement est gardé constant et égal à 3,62, seul celui de la couche active varie. On peut noter l'augmentation de $\theta_{\perp}$ avec $\mathrm{n}_{\text {act' }}$ c'est-à-dire plus la valeur de l'indice de la couche active est élevée, plus grande sera celle de $\theta_{\perp}$. 
Afrique SCIENCE 05(1)(2009) 58 - 66

II apparaît que l'augmentation de la concentration en Aluminium des couches de confinement permet de réduire nettement l'indice de réfraction comme illustré dans le tableau 1.

Par ailleurs, Adams et Cross [18] ont montré sur les DH lasers $G a_{1-x} A l_{x} A s / G a A s / G a_{l}$. ${ }_{x} A l_{x} A s$, que la perte par diffraction est plus élevée pour les valeurs de $x$ comprises entre 0 et 0,1 et faible pour des valeurs élevées de la composition. Ce qui d'après les travaux effectués par Clark et al [19], résulterait de la séparation spin-orbite plus élevée aux faibles valeurs $x$ de la composition qui est à l'origine de l'augmentation de l'absorption dans les alliages formant les couches de confinement.

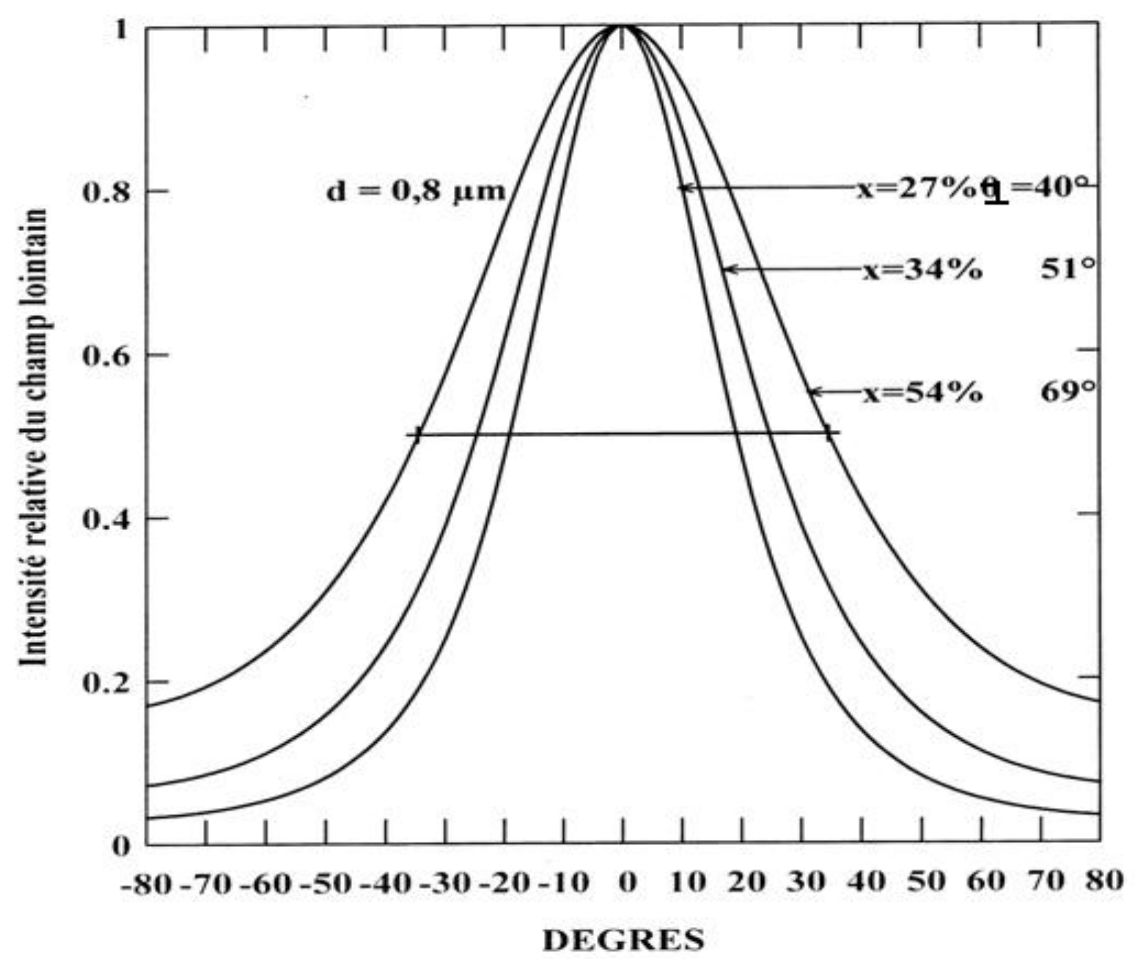

Figure 2 : Influence de la composition x d'Aluminium sur la valeur de l'écart des angles à mi-hauteur $\theta_{\perp}$ de l'intensité du champ lointain. 
65

Afrique SCIENCE 05(1)(2009) $58-66$

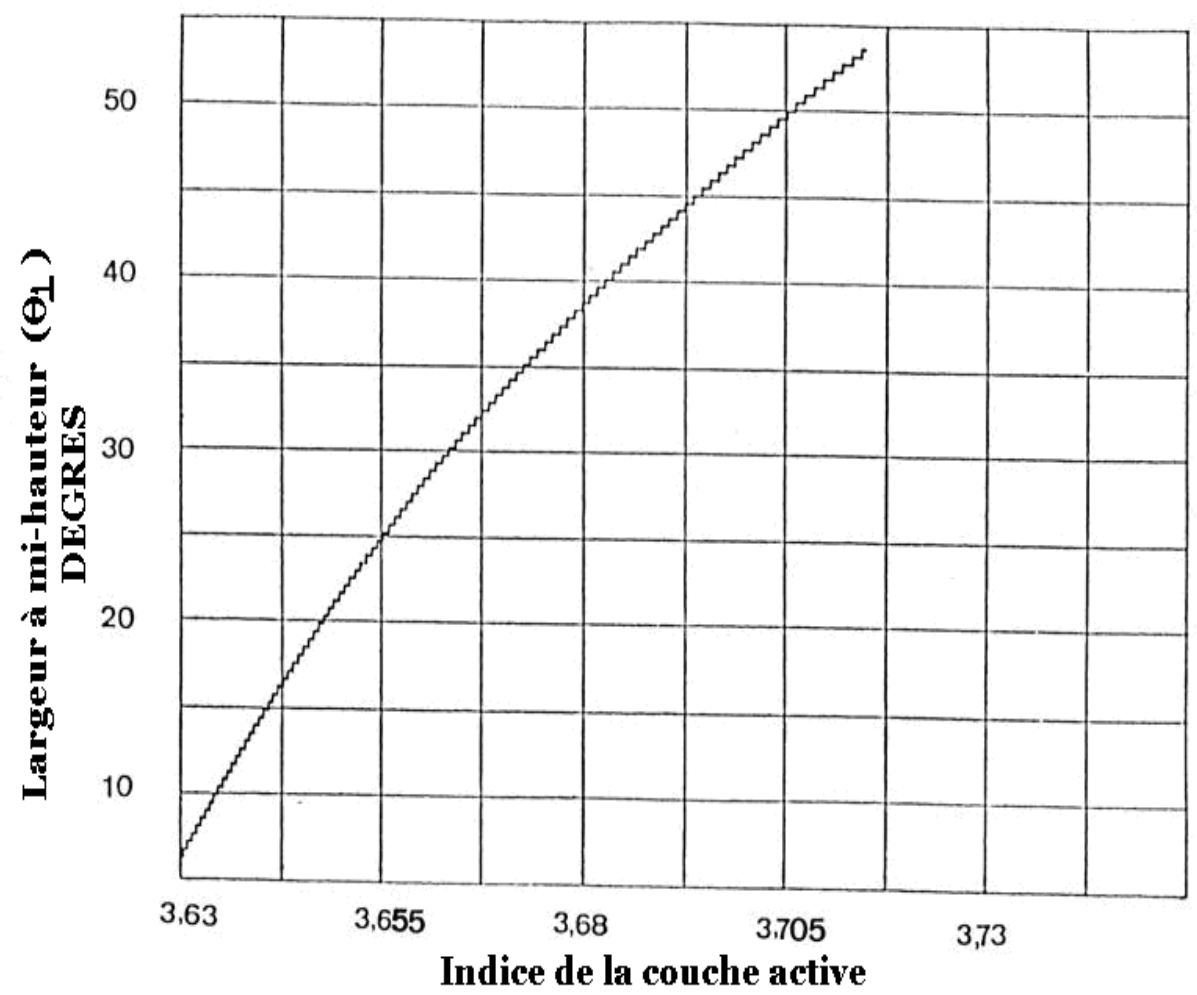

Figure 3 : Largeur à mi-hauteur $\left(\theta_{\perp}\right)$ en fonction de l'indice de la couche active. L'indice de la couche de confinement est gardé constant et vaut 3,62

\section{Conclusion}

D'une manière générale, la distribution de l'intensité lumineuse du champ lointain d'une double hétérostructure laser dépend de l'indice de réfraction $\mathrm{n}_{\text {act }}$ de la couche active et de $\mathrm{n}_{\text {conf }}$ des couches de confinement.

L'écart $\Delta \mathrm{n}=\mathrm{n}_{\text {act }}-\mathrm{n}_{\text {conf }}$ est caractéristique de la structure guidante; ainsi toute variation de $\Delta \mathrm{n}$ peut modifier les propriétés de la couche guidante.

Il est donc nécessaire de connaître avec précision la variation de l'indice de réfraction avec la composition $\mathrm{x}$ d'Aluminium et $\mathrm{x}$ ' d'Indium des quaternaires $G a_{1-x} A l_{x} A s_{y} S b_{1-y}$ ef $G a_{1-x} / n_{x} A s_{y} S b_{1-y^{*}}$ 
Afrique SCIENCE 05(1)(2009) $58-66$

Les travaux effectués par CANEAU et al.[20] sur des structures similaires aux nôtres montrent qu'on peut avoir des densités de courant de seuil typiques de 7 à $8 \mathrm{KA} / \mathrm{cm}^{2}$ à la température ambiante, pour des valeurs de $\mathrm{d}$ comprises entre 0,8 ef $1 \mu \mathrm{m}$.

\section{Références}

[1] - M.A. MOHOU, IE (I) Journal- ET, Vol 76(1996) 81-85.

[2] - H. K. CHOI, J. N. WALPOLE, G. W. TURNER, S. J. EGLASH, L. S. MISSIGIA and M. K. CONNORS, IEEE photonics Technology Letters, Vol 5 (1993) 1117.

[3] - A. N. BARANOV, S. Yu, BELKIN, T. N. DANILOVA, O. G. ERSHOV, A. N. IMENKOV and Yu P. YAKOVLEV, Soviet Physics Semiconductors, Vol 26 ( 1992) 1107.

[4] - H. K. CHOI, G. W. TURNER and S. J. EGLASH, IEEE photonics Technology Letters, Vol 6 ( 1994) 7.

[5] - H. K. CHOI, and S. J. EGLASH, Applied Physics Letters, Vol 61 (1992) 1154.

[6] - D. MARCUSE, "Integrated Optics", Ed. by Van Nostrand Reinhold, New York (1972)

[7] - P. M. ASBECK, D. A. CAMMACK, J.J. DANIELE and V. KLEBANOFF, IEEE J. Quantum Electron., Vo/15 (1979) 727-733

[8] - H. C. CASEY Jr. and M.B. PANISH, "Heterostructure lasers, Part A" Academic Press, New york, (1978)

[9] - BORN and WOLF, "Principle of optics" Ed. by Pregamon Press, London(1970)

[10] - H. C. CASEY Jr., J. Appl. Phys., Vol 49(1978) 3678-3692

[11] - M. YANO, H. NISHI and M. TAKUSAGAWA, IEEE J. Quantum Electron, Vo/ 15 (1979) 571-579

[12] - G. A. HOCKHAM, Electron. lett., Vol 9(1973) 389-391

[13] - D. BOTEZ,R.C.A. Review, Vol 39(1978) 577-603

[14] - ]. MATHEWS and R.L. WALKER, , Ed. by W. A. BENJAMIN, Inc.,New York (1970) 82-90

[15] - N. E. BYER and J.K. BUTLER, IEEE J. Quantum Electron, Vol 6 (1970) 291-296

[16] - J.K. BUTLER and J. ZOROOFCHI, IEEE J. Quantum Electron., Vol (1974) 809-815

[17] - Y. ITAYA, Y. SUEMATSU, S. KATAYAMA, K. KISHINO and S. ARAI, Jpn,J. Appl. Phys., Vol 18 (1979) 1795-1805

[18] - M. J. ADAMS and M. CROSS, Solid. State Electron., Vol 14 (1971) 865-883

[19] - G. D. CLARK Jr. and N. HOLONYAK Jr., Phys. Rev., Vol 156 (1967) 913-924

[20] - C.CANEAU, A.K. SRIVASTAVA, A.G. DENTAI, J.L. ZYSKIND, C.A. BURRUS and M.A. POLLACK, Electron. Lett., Vol 22 (1986) 992-993. 